\title{
Wave Propagation in a Two Component Warm Plasma
}

\author{
S. R. Seshadri \\ Applied Research Laboratory, Sylvania Electronic Systems, a Division of Sylvania Electric \\ Products, Inc., Waltham, Mass., 02154
}

(Received August 20, 1964; revised November 20, 1964)

\begin{abstract}
A treatment of the characteristics of a plane wave propagating in a homogeneous, unbounded, and fully ionized plasma is given by employing the linearized, two-fluid continuum theory of plasma dynamics. The plasma is assumed to be a macroscopically neutral and loss-free mixture of gas of electrons and singly charged ions. The dispersion relations for the general case of propagation at an arbitrary angle to the direction of the static magnetic field are investigated without any limitation on the frequency and with emphasis on the coupling of the transverse and the longitudinal type waves. Simple analytical expressions for the dispersion relations in the various intervals of frequency and phase velocity, as well as for the frequencies where the transverse and the longitudinal type waves couple, are given in terms of the various parameters. This treatment does not cover the cases in which the propagation vector is in the close neighborhood of either the direction of the static magnetic field or that perpendicular to it.
\end{abstract}

\section{Introduction}

By far the largest number of applications to the study of the nature of wave propagation in the ionosphere are based on the magneto-ionic theory of Appleton and Hartree [Ratcliffe, 1959]. This theory is concerned with the treatment of the plane wave dispersion relations in a cold, homogeneous and unbounded electron plasma. A number of extensions to the theory of Appleton and Hartree are available in the literature. The extension of the conventional magneto-ionic theory to the low frequencies by the inclusion of the motion of the heavy ions has been investigated by various authors [Astrom, 1950, 1951; Stix, 1957; Fejer, 1960; Booker, 1963; Story, 1956; Hines, 1953, 1957, 1963; Seshadri, 1964a]. The magneto-ionic theory together with its hydromagnetic extension obtained by taking into account the motion of the heavy ions has been able to predict correctly the nature of the plane wave propagation in a plasma, under the circumstances in which the effects of the alternating electric field predominate over those of the pressure gradients.

When the effects of the pressure gradients are not negligible, the magneto-ionic theory fails to provide an adequate description of the properties of wave motion in a plasma. Also when the pressures of the charged particles are taken into account, new types of wave motion are found to appear. There have been a number of investigations on the plane wave dispersion relations in a compressible plasma which is capable of sustaining not only the usual transverse electromagnetic type waves but also the longitudinal plasma waves. These investigations are all based on a linearized theory of plasma oscillations and the essentials of this theory have been summarized in a recent paper by Oster [1960]. The characteristics of a plane wave propagating in an unbounded single component warm plasma have been treated [Oster, 1960] only for the two special cases for which the propagation vector is either along or across the direction of the external magnetostatic field. Recently a systematic development of the theory of plane wave propagation in an unbounded, compressible electron plasma has been given for the general case in which the propagation vector makes an arbitrary angle with the direction of the magnetostatic field [Kieburtz, 1964; Seshadri, $1964 \mathrm{a}$ and $\mathrm{b}]$. 
The plane wave dispersion relations in a compressible plasma wherein motions of both the electrons and the ions are taken into account have also been studied by Pai [1960, 1962], Watanabe [1961], and Tanenbaum [1961]. The treatments of these authors pertain to the case with no applied magnetic field or to simpler cases of propagation parallel or perpendicular to the direction of the external magnetic field. Various simpler aspects and special cases of the plane wave dispersion relations in a two component, warm plasma are contained in the books by Stix [1962], Allis, Buchsbaum, and Bers [1963] and Denisse and Delcroix [1963].

In this paper, the plane wave dispersion relations in a two component warm magnetoplasma are investigated in a systematic manner. The dispersion equation, which is essentially a quartic, is derived without making any approximations in the beginning and is cast in a form amenable to analysis by a perturbation procedure. As a natural consequence of the procedure employed in analyzing the dispersion relations, the coupling regions where the high phase velocity, predominantly transverse waves merge with those of the low phase velocity, predominantly longitudinal waves become evident. Moreover, simple analytical expressions for various sections of the dispersion curves are obtained and this enables the proper understanding of the changes in the dispersion caused by the variations of the relevant parameters.

It is only appropriate to mention the reasons for using the continuum theory in the present investigation. Basically, there are two methods of dealing with the plasma problems, namely the kinetic treatment based on the Boltzmann equation and the hydrodynamic treatment based on the various transport equations. The first method is more accurate but leads to greater mathematical complexities. The second method, though incapable of accounting for effects such as Landau damping, is more readily amenable to analysis. Moreover, the macroscopic treatment gives correctly the various waves and their phase velocities and this knowledge is bound to provide valuable guidelines for the more complete treatments based on the Boltzmann equation.

\section{Statement of the Problem}

Consider a homogeneous plasma of infinite extent. The plasma is idealized to be a lossless and macroscopically neutral mixture of gas of electrons and a single species of ions. The drift velocity of the electrons and the ions are assumed to be zero so that the plasma, as a whole, may be considered to be stationary. A uniform magnetic field $B_{0}$ is assumed to be impressed externally throughout the plasma in the $z$-direction, where $x, y$, and $z$ form a right-handed rectangular coordinate system (fig. 1). It is proposed to restrict attention only to the linear, time-harmonic problem; the harmonic time dependence of the form $e^{-i \omega t}$ is implied for all the field components.

Let $N_{e}, \overrightarrow{V_{e}}$, and $P_{e}$ be respectively, the average number density, the velocity and the pressure of the electrons; and let $N_{i}, \vec{V}_{i}$, and $P_{i}$ be the corresponding quantities for the ions. Also let $\vec{E}$ and $\vec{H}$ be the alternating electric and magnetic fields. The linearized time-harmonic hydrodynamic equations of motion for the electrons and the ions are

$$
\begin{aligned}
& -i \omega m_{e} N_{e} \vec{V}_{e}=-N_{e} e\left(\vec{E}+\overrightarrow{V_{e}} \times \hat{z} B_{0}\right)-\nabla P_{e} \\
& -i \omega m_{i} N_{i} \vec{V}_{i}=N_{i} e\left(\vec{E}+\vec{V}_{i} \times \hat{z} B_{0}\right)-\nabla P_{i}
\end{aligned}
$$

where $-e$ and $m_{e}$ are respectively the charge and the mass of an electron and $+e$ and $m_{i}$ are the corresponding quantities for an ion. Since the plasma is assumed to be neutral, the number densities of the electrons and the ions are equal and hence, each may be set equal to $N_{0}$, for convenience. The linearized equations of continuity combined with the equations of state are given by

$$
\begin{aligned}
& u_{e}^{2} m_{e} N_{0} \nabla \cdot \vec{V}_{e}=i \omega P_{e} \\
& u_{i}^{2} m_{i} N_{0} \nabla \cdot \vec{V}_{i}=i \omega P_{i}
\end{aligned}
$$


FigURE 1. Geometry of the problem.

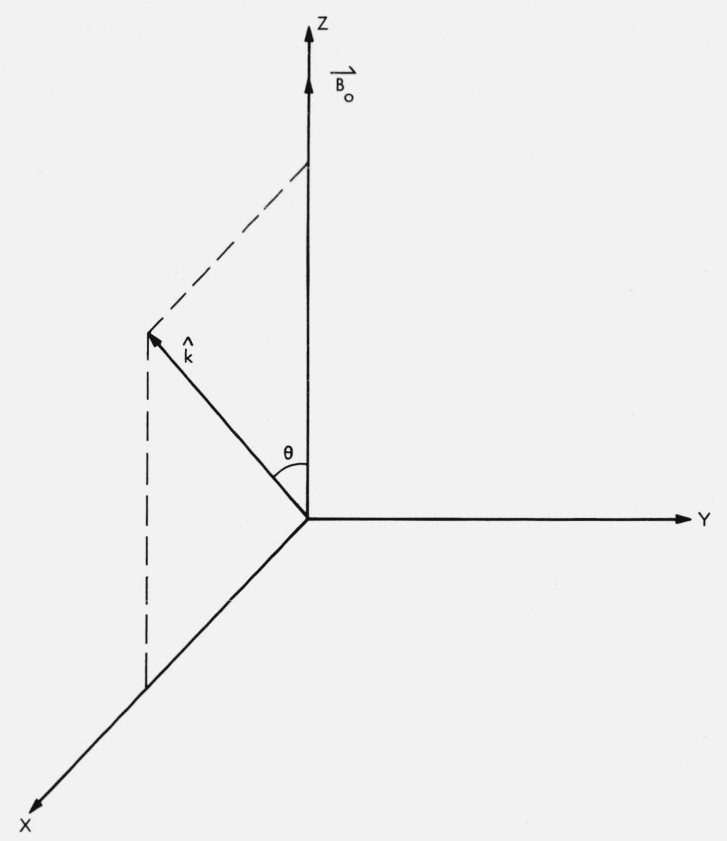

where $u_{e}$ and $u_{i}$ are the sound velocities in the electron gas and the ion gas respectively. In addition, the electric and the magnetic fields satisfy the following time-harmonic Maxwell's equations

$$
\begin{aligned}
& \nabla \times \vec{E}=i \omega \mu_{0} \vec{H} \\
& \nabla \times \vec{H}=-i \omega \epsilon_{0} \vec{E}+N_{0} e\left(\vec{V}_{i}-\vec{V}_{e}\right)
\end{aligned}
$$

where $\mu_{0}$ and $\epsilon_{0}$ are respectively the permeability and the dielectric constant of free space.

It is desired to investigate the characteristics of a plane wave propagating in the plasma medium. Let $\vec{k}$ be the propagation vector such that $\hat{z} x \vec{k}$ coincides with the $y$-axis and let $\theta$ be the angle that $\vec{k}$ makes with the direction of the static magnetic field, so that $k_{x}=k \sin \theta=k n, k_{y}=0$ and $k_{z}=k \cos \theta=k l$. Therefore, all the field components will have the spatial dependence of the form $e^{i k(n x+l z)}$ so that

$$
\frac{\partial}{\partial x}=i k n ; \frac{\partial}{\partial y}=0 ; \frac{\partial}{\partial z}=i k l \text {. }
$$

On eliminating $P_{e}$ from (1) with the help of (3) and (7), an expression for $\vec{V}_{e}$ can be obtained in terms of $\vec{E}$. In a similar manner, the elimination of $P_{i}$ from (2) with the help of (4) and (7) leads to an expression for $\overrightarrow{V_{i}}$ in terms of $\vec{E}$. On substituting (5), (7), and the expressions for $\vec{V}_{e}$ and $\vec{V}_{i}$ in terms of $\vec{E}$ into (6), the following set of equations specifying $E_{x}, E_{y}$, and $E_{z}$ is obtained.

$$
\left[\begin{array}{rrr}
D_{11} & i D_{12} & D_{13} \\
-i D_{21} & D_{22} & -i D_{23} \\
D_{31} & i D_{32} & D_{33}
\end{array}\right]\left[\begin{array}{l}
E_{x} \\
E_{y} \\
E_{z}
\end{array}\right]=[D][E]=0
$$

where

$$
\begin{aligned}
& D_{11}=\frac{k^{2} l^{2} C_{0}^{2}}{\omega^{2}}-1+\frac{\omega_{p e}^{2}}{\omega^{2} \alpha_{e} D_{e}}\left(1-\frac{k^{2} u_{e}^{2} l^{2}}{\omega^{2}}\right)+\frac{\omega_{p i}^{2}}{\omega^{2} \alpha_{i} D_{i}}\left(1-\frac{k^{2} u_{i}^{2} l^{2}}{\omega^{2}}\right) \\
& D_{12}=D_{21}=-\frac{\omega_{p e}^{2} \omega_{c e}}{\omega^{3} \alpha_{e} D_{e}}\left(1-\frac{k^{2} u_{e}^{2} l^{2}}{\omega^{2}}\right)+\frac{\omega_{p i}^{2} \omega_{c i}}{\omega^{3} \alpha_{i} D_{i}}\left(1-\frac{k^{2} u_{i}^{2} l^{2}}{\omega^{2}}\right)
\end{aligned}
$$




$$
\begin{aligned}
& D_{13}=D_{31}=-\frac{k^{2} l n C_{0}^{2}}{\omega^{2}}+\frac{\omega_{p e}^{2}}{\omega^{2} \alpha_{e} D_{e}} \frac{k^{2} u_{e}^{2} l n}{\omega^{2}}+\frac{\omega_{p i}^{2}}{\omega^{2} \alpha_{i} D_{i}} \frac{k^{2} u_{i}^{2} l n}{\omega^{2}} \\
& D_{22}=\frac{k^{2} C_{0}^{2}}{\omega^{2}}-1+\frac{\omega_{p e}^{2}}{\omega^{2} \alpha_{e} D_{e}}\left(1-\frac{k^{2} u_{e}^{2}}{\omega^{2}}\right)+\frac{\omega_{p i}^{2}}{\omega^{2} \alpha_{i} D_{i}}\left(1-\frac{k^{2} u_{i}^{2}}{\omega^{2}}\right) \\
& D_{23}=D_{32}=-\frac{\omega_{p e}^{2} \omega_{c e}}{\omega^{3} \alpha_{e} D_{e}} \frac{k^{2} u_{e}^{2} l n}{\omega^{2}}+\frac{\omega_{p i}^{2} \omega_{c i}}{\omega^{3} \alpha_{i} D_{i}} \frac{k^{2} u_{i}^{2} l n}{\omega^{2}} \\
& D_{33}=\frac{k^{2} n^{2} C_{0}^{2}}{\omega^{2}}-1+\frac{\omega_{p e}^{2}}{\omega^{2} D_{e}}\left(1-\frac{k^{2} u_{e}^{2} n^{2}}{\omega^{2} \alpha_{e}}\right)+\frac{\omega_{p i}^{2}}{\omega^{2} \alpha_{e}}\left(1-\frac{k^{2} u_{i}^{2} n^{2}}{\omega^{2} \alpha_{i}}\right) \\
& D_{n}=1-\frac{k^{2} u_{n}^{2}}{\omega^{2} \alpha_{n}}\left(1-l^{2} \frac{\omega_{c n}^{2}}{\omega^{2}}\right) ; \alpha_{n}=1-\frac{\omega_{c n}^{2}}{\omega^{2}} \quad n=e, i .
\end{aligned}
$$

Also $\omega_{p e}\left(\omega_{p i}\right)$ and $\omega_{c e}\left(\omega_{c i}\right)$ are the plasma and the gyromagnetic frequency of the electrons (ions) and are given by

$$
\omega_{p n}^{2}=\frac{N_{0} e^{2}}{m_{n} \epsilon_{0}} ; \omega_{c n}=\frac{e B_{0}}{m_{n}} \quad n=e, i
$$

and $C_{0}=1 / \sqrt{\mu_{0} \epsilon_{0}}$ is the free space electromagnetic wave velocity. The determinant of $[D]$ should be equal to zero in order that (8) may have a nontrivial solution and this condition leads to the dispersion equation.

The two special cases of propagation along and across the static magnetic field are reviewed briefly before proceeding to the study of the dispersion relations for the arbitrary direction of propagation.

\section{Propagation Along the Direction of the Static Magnetic Field}

For the case of propagation along the direction of the static magnetic field, $l=1$ and $n=0$. Therefore, $\epsilon_{13}=\epsilon_{31}=\epsilon_{23}=\epsilon_{32}=D_{13}=D_{31}=D_{23}=D_{32}=0$ and $\epsilon_{11}=\epsilon_{22}, D_{11}=D_{22}$ and $D_{33}=-\epsilon_{33}$. Consequently the general dispersion equation factors into the following three separate equations:

$$
D_{11}-D_{12}=0 ; \quad D_{11}+D_{12}=0 ; \quad D_{33}=-\epsilon_{33}=0 .
$$

With the help of (9), (12a, b) may be shown to yield the following expressions:

$$
V=C_{0}\left(\epsilon_{1}+\epsilon_{2}\right)^{-1 / 2} ; \quad V=C_{0}\left(\epsilon_{1}-\epsilon_{2}\right)^{-1 / 2}
$$

where $V=\frac{\omega}{k}$ is the phase velocity of the wave and

$$
\begin{aligned}
& \epsilon_{1}=1-\frac{1}{\Omega^{2}-R^{2}}-\frac{m}{\Omega^{2}-R^{2} m^{2}} \\
& \epsilon_{2}=\frac{R}{\Omega\left(\Omega^{2}-R^{2}\right)}-\frac{R m^{2}}{\Omega\left(\Omega^{2}-R^{2} m^{2}\right)}
\end{aligned}
$$

and

$$
\Omega=\frac{\omega}{\omega_{p e}} ; \quad R=\frac{\omega_{c e}}{\omega_{p e}} ; \quad m=\frac{m_{e}}{m_{i}} .
$$


In this investigation $m_{e} / m_{i}$ will be set equal to $1 / 1836$ which corresponds to a hydrogen plasma. In general, $m$ is very much small in comparison with unity. It is to be noted that $\Omega$ is the normalized frequency and $R$ is the normalized strength of the external magnetic field. The substitution of (14a) and (14b) in (13a) and (13b) yields

$$
\begin{aligned}
& V_{\mathrm{EM}(0)}=C_{0}\left[\frac{(\Omega+R)(\Omega-R m)}{\Omega^{2}+\Omega R(1-m)-\left(1+m+R^{2} m\right)}\right]^{1 / 2} \\
& V_{\mathrm{EM}(e)}=C_{0}\left[\frac{(\Omega-R)(\Omega+R m)}{\Omega^{2}-\Omega R(1-m)-\left(1+m+R^{2} m\right)}\right]^{1 / 2}
\end{aligned}
$$

Note that in the phase velocities (15a) for the ordinary mode and (15b) for the extraordinary modes neither of the two acoustic velocities $u_{e}$ and $u_{i}$ appear. Evidently therefore, $(15 \mathrm{a}, \mathrm{b})$ pertain to the purely transverse electromagnetic modes with no coupling to the longitudinal plasma waves. These two modes are the same as in an incompressible plasma for the case of propagation along the static magnetic field and have been studied previously [Seshadri, 1964a]. In figures $2 \mathrm{a}$ and $\mathrm{b}$ the phase velocities given by $(15 \mathrm{a}, \mathrm{b})$ are plotted for two values of $R^{2}$, namely $R^{2}=1 / 2$ and $R^{2}=4 / 3$.

In a similar manner, it may be easily shown with the help of (9) that (12c) yields

$$
\left(\Omega^{2}-1-m\right) V^{4}-\left[\Omega^{2} u_{e}^{2}+m u_{e}^{2}\left(\Omega^{2}-2\right)\right] V^{2}+\Omega^{2} m u_{e}^{4}=0 .
$$

The dispersion equation (16) which does not contain the velocity $C_{0}$ of electromagnetic waves in free space obviously corresponds to the purely longitudinal plasma waves with no coupling to the purely transverse electromagnetic waves. Also (16) does not depend on the static magnetic field, as is to be expected since the purely longitudinal plasma waves are unaffected by the static mag. netic field for the case of propagation parallel to its direction. The study of the dispersion equation (16) has shown [Seshadri, 1965] that the electron plasma (EP) mode propagates for $\Omega>1$ and its phase velocity approaches $u_{e}$ in the limit of infinite frequency. On the other hand, the ion plasma

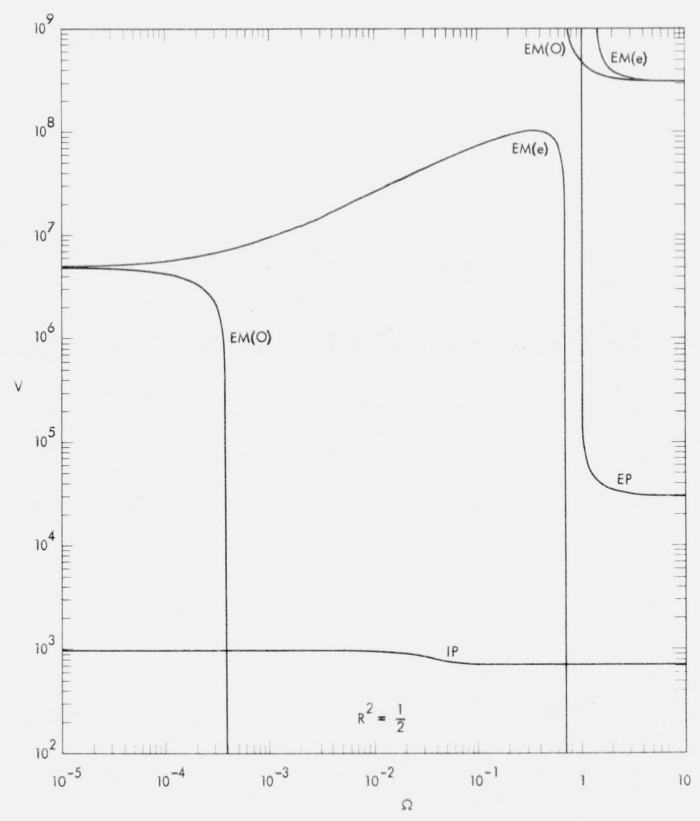

FIGURE 2a. Dispersion relations for propagation along the static magnetic field $\left(\mathrm{R}^{2}=\frac{1}{2}\right)$.

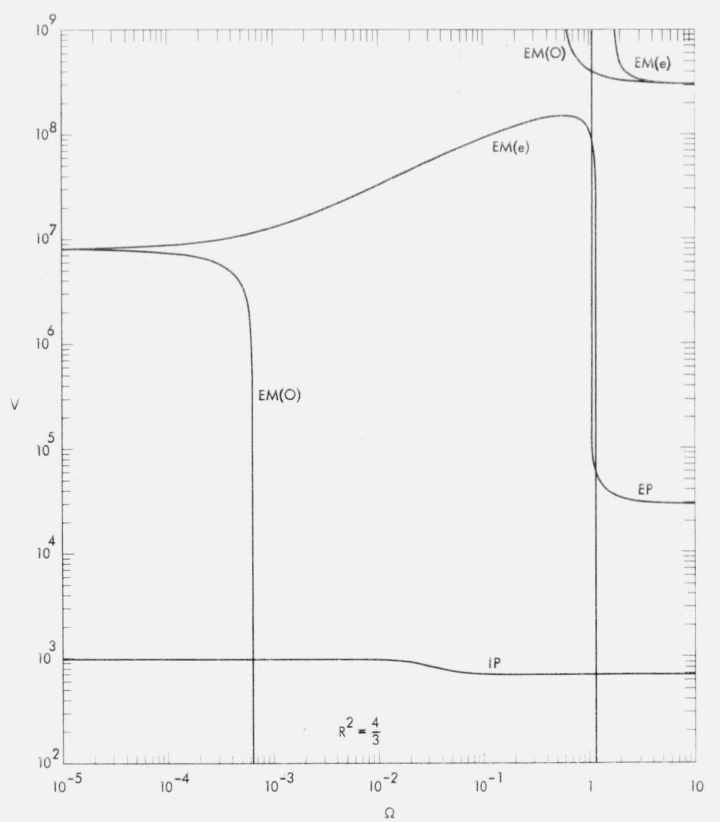

FIGURE 2b. Dispersion relations for propagation along the static magnetic field $\left(\mathrm{R}^{2}=\frac{4}{3}\right)$. 
(IP) mode propagates for all frequencies and its phase velocity approaches $u_{i}$ in the limit of infinite frequency and the velocity $\sqrt{2} u_{i}$, which is the velocity of the acoustic waves in the whole gas of charged particles, in the limit of zero frequency. In fact, the transition of the phase velocity of the ion plasma mode from the value $\sqrt{2} u_{i}$ to the value $u_{i}$ takes place rather rapidly near the frequency given by $\Omega^{2}=2 m$. The phase velocities of the electron and the ion plasma modes, as given by the dispersion equation (16) are also plotted in figures $2 \mathrm{a}$ and $2 \mathrm{~b}$. The values of $C_{0}$ and $u_{e}$ are taken to be equal to $3 \times 10^{8}$ and $3 \times 10^{4} \mathrm{~m} / \mathrm{sec}$ respectively.

\section{Propagation Across the Direction of the Static Magnetic Field}

For the case of propagation across the direction of the static magnetic field, $l=0$ and $n=1$. Therefore, as before, $\epsilon_{13}=\epsilon_{31}=\epsilon_{23}=\epsilon_{32}=D_{13}=D_{31}=D_{23}=D_{32}=0$. Consequently the general dispersion equation factors into the following two separate equations:

$$
D_{11} D_{22}-D_{12}^{2}=0 ; D_{33}=0 \text {. }
$$

With the help of (9), (17b) may be shown to yield

$$
V_{\mathrm{EM}(0)}=C_{0} \Omega\left(\Omega^{2}-1-m\right)^{-1 / 2} .
$$

Since neither of the two acoustic velocities, $u_{e}$ and $u_{i}$ appear, (18) evidently corresponds to a purely transverse electromagnetic wave without any coupling to the longitudinal plasma waves. Also (18), which corresponds to the ordinary electromagnetic mode as indicated by the subscript $\operatorname{EM}(0)$ on $V$, is independent of the static magnetic field. As the ordinary electromagnetic mode has its electric vector in the direction of the static magnetic field, which exerts no force parallel to itself, its dispersion is unaffected by the static magnetic field for the case of propagation across its direction. The phase velocity given by (18) is plotted in figure 3 .

With the help of (9) and (10), (17a) may be shown to yield the following cubic equation in $V^{2}$ :

$$
\begin{gathered}
A_{0} V^{6}+A_{1} V^{4}+A_{2} V^{2}+A_{3}=0 \\
A_{0}=\Omega^{4}-\Omega^{2}\left(2+2 m+R^{2}+R^{2} m^{2}\right)+\left(1+m+R^{2} m\right)^{2} \\
A_{1}=-C_{0}^{2}\left[\Omega^{4}(1+\delta+m \delta)-\Omega^{2}\left\{1+m+\delta+4 m \delta+m^{2} \delta+R^{2}\left(1+m^{2}\right.\right.\right. \\
\left.\left.\left.+m \delta+m^{2} \delta\right)\right\}+2 m \delta(1+m)+R^{2} m\left(1+m+2 m \delta+R^{2} m\right)\right] \\
A_{2}=\Omega^{2} C_{0}^{2} u_{e}^{2}\left[\Omega^{2}(1+m+m \delta)-m\left(2+\delta+m \delta+R^{2}+R^{2} m\right)\right] \\
A_{3}=-\Omega^{4} m u_{e}^{4} C_{0}^{2}
\end{gathered}
$$

when

$$
\delta=u_{e}^{2} / C_{0}^{2}
$$

The dispersion equation (19) which contains $C_{0}, u_{e}$, and $m$, corresponds to modes that have transverse and longitudinal field components and in which the motion of both the electrons and the ions contribute to the wave propagation. It is possible to decompose the total field into three independent modes which have been designated as (i) the modified extraordinary electromagnetic mode $[\mathrm{MEM}(\mathrm{x})]$, (ii) the modified electron plasma mode [MEP], and (iii) the modified ion plasma mode [MIP]. The phase velocity of these three modes are given by (19), which is solved by a 


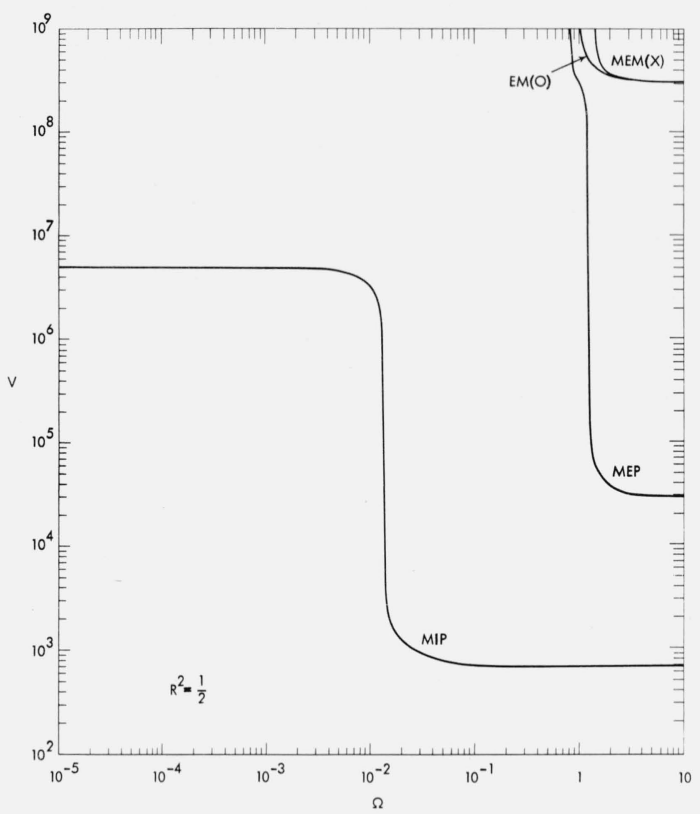

FIGURE 3. Dispersion relations for propagation across the static magnetic field $\left(\mathrm{R}^{2}=\frac{1}{2}\right)$.

method similar to the one detailed in the next section and the following result is obtained:

$$
\begin{aligned}
V_{\mathrm{MEM}(x)} & =C_{0}\left[\frac{\Omega^{2}\left(\Omega^{2}-1-R^{2}\right)}{\Omega^{4}-\Omega^{2}\left(R^{2}+2\right)+1}\right]^{1 / 2} \text { for } \Omega>\Omega_{2} \\
V_{\mathrm{MEP}} & =C_{0}\left[\frac{\Omega^{2}\left(\Omega^{2}-1-R^{2}\right)}{\Omega^{4}-\Omega^{2}\left(R^{2}+2\right)+1}\right]^{1 / 2} \text { for } \Omega_{1}<\Omega<\sqrt{1+R^{2}} \\
& =\Omega \sqrt{C_{0} u_{e}}\left[\frac{1}{R}\left\{1+\frac{C_{0}^{2}}{u_{e}^{2}} \frac{\left(\Omega^{2}-1-R^{2}\right)}{4 R^{2}}\right\}^{1 / 2}-\frac{\left(\Omega^{2}-1-R^{2}\right)}{2 R^{2}} \frac{C_{0}}{u_{e}}\right]^{1 / 2} \\
& =u_{e} \Omega\left(\Omega^{2}-1-R^{2}\right)^{-1 / 2} \text { for } \sqrt{1+R^{2}}<\Omega<\infty \\
V_{\mathrm{MIP}} & =C_{0}\left[R^{2} m-\Omega^{2}\left(1+R^{2}\right]^{1 / 2} \text { for } 0<\Omega^{2}-1-R^{2} \mid \leqslant \frac{u_{e}}{C_{0}}\right. \\
& \left.=\left[-\frac{C_{0}^{2}\left\{\Omega^{2}\left(1+R^{2}\right)-R^{2} m\right\}+\frac{1}{2}\left[C_{0}^{4}\left\{\Omega^{2}\left(1+R^{2}\right)-R^{2} m\right\}^{2}\right.}{2} \quad-4 \Omega^{2} C_{0}^{2} u_{e}^{2}\left\{\Omega^{2}-m\left(2+R^{2}\right)\right\}\right]^{1 / 2}\right]^{1 / 2} \text { for }\left|\Omega^{2}-\Omega_{\alpha}^{2}\right| \leqslant \frac{u_{e}}{C_{0}} m \\
& \left.\left.\left.+\Omega_{e}\left[\frac{m\left(2+R^{2}\right)-\Omega^{2}}{\Omega^{2}\left(1+R^{2}\right)-R^{2} m}\right]^{1 / 2} \text { for } \Omega_{\alpha}<\Omega_{e}^{4}<\Omega_{0}^{2}-m\left(2+R^{2}\right)\right\}+4 m \Omega^{4} u_{e}^{4}\left\{\Omega^{2}\left(1+R^{2}\right)-R^{2} m\right\}\right]^{1 / 2}\right]^{1 / 2} \\
& =\left\{\Omega^{2}\left(1+R^{2}\right)-R^{2} m\right\}^{-1 / 2}\left[-\frac{\Omega^{2} u_{e}^{2}}{2}\left\{\Omega^{2}-m\left(2+R^{2}\right)\right\}\right. \\
& \text { for }\left|\Omega_{0}^{2}\right| \leqslant m
\end{aligned}
$$

$=\Omega \sqrt{m} u_{e}\left[\Omega^{2}-m\left(2+R^{2}\right)\right]^{-1 / 2}$ for $\Omega_{0}<\Omega<\infty$ 
where

$$
\begin{aligned}
\Omega_{1,2} & =\mp \frac{R}{2}+\sqrt{\left(\frac{R}{2}\right)^{2}+1} \\
\Omega_{\alpha} & =\left(\frac{R^{2} m}{1+R^{2}}\right)^{1 / 2} ; \quad \Omega_{0}=\left[m\left(2+R^{2}\right)\right]^{1 / 2}
\end{aligned}
$$

In obtaining (21) - (23), it has been assumed that

$$
C_{0}^{2}>>V_{\alpha}^{2}>>U_{a}^{2}
$$

where $V_{\alpha}=C_{0} R \sqrt{m}$ is the Alfvén wave velocity and $U_{a}=\sqrt{2 m} u_{e}$ is the acoustic velocity in the entire gas of charged particles. The condition (25) is usually obtained im most practical cases and corresponds to the value of $R^{2}$ being of the order of unity.

Although, as stated earlier, the three modes corresponding to (19) have both longitudinal and transverse field components, it may be easily verified that (i) the modified extraordinary electromagnetic mode is predominantly transverse throughout its frequency range of propagation, $\Omega_{2}<\Omega<\infty$, (ii) the modified electron plasma mode is predominantly transverse for $\Omega_{1}<\Omega<\sqrt{1+R^{2}}$ and is predominantly longitudinal for $\sqrt{1+R^{2}}<\Omega<\infty$, and (iii) the modified ion plasma mode is predominantly transverse for $0<\Omega<\Omega_{\alpha}$ and is predominantly longitudinal for $\Omega_{\alpha}<\Omega<\infty$.

In the limit of infinite frequency, the phase velocities of the $\operatorname{MEM}(x)$, MEP and MIP modes approach $C_{0}, u_{e}$, and $u_{i}$ respectively. Also in the limit of zero frequency, the phase velocity of the MIP mode approaches $V_{\alpha}$. The phase velocities of the three modes given by (21)-(23) are plotted in figure 3 for $R^{2}=1 / 2$.

\section{Propagation in an Arbitrary Direction With Respect to That of the Static Magnetic Field}

For arbitrary direction of propagation, the determinant of $[D]$ does not factor into two simpler equations and this makes the analysis of the dispersion relations for the general case very complicated. With the help of (8), (9), and (10), the following quartic equation in $V^{2}$ is obtained:

$$
A_{0} V^{8}+A_{1} V^{6}+A_{2} V^{4}+A_{3} V^{2}+A_{4}=0
$$

where

$$
\begin{aligned}
A_{0}=\left(\Omega^{2}-1-\right. & m)\left[\Omega^{4}-\Omega^{2}\left(2+2 m+R^{2}+R^{2} m^{2}\right)+\left(1+2 m+2 R^{2} m+m^{2}+2 R^{2} m^{2}+R^{4} m^{2}\right)\right] \\
A_{1}=C_{0}^{2}\left[-\Omega^{6}(2+\delta+\right. & m \delta)+\Omega^{4}\left\{\left(4+4 m+2 \delta+6 m \delta+2 m^{2} \delta\right)\right. \\
& \left.+R^{2}\left(2+2 m^{2}+\delta l^{2}+m \delta+m^{2} \delta+m^{3} \delta l^{2}\right)\right\}-\Omega^{2}\{(2+4 m \\
& \left.+2 m^{2}+\delta+7 m \delta+7 m^{2} \delta+m^{3} \delta\right)+R^{2}\left(1+l^{2}+4 m+4 m^{2}\right. \\
& \left.+m^{3}\left(1+l^{2}\right)+3 m \delta l^{2}+m \delta+4 m^{2} \delta+m^{3} \delta+3 m^{3} \delta l^{2}\right) \\
& \left.+R^{4} m^{2}\left(2+\delta l^{2}+m \delta l^{2}\right)\right\}+2 m \delta\left(1+2 m+m^{2}\right)+R^{2} m\left(1+l^{2}\right. \\
& +2 m+2 m l^{2}+m^{2}\left(1+l^{2}\right)+2 m \delta+2 m \delta l^{2}+2 m^{2} \delta \\
& \left.\left.+2 m^{2} \delta l^{2}\right)+R^{4} m^{2}\left(1+l^{2}+m+m l^{2}+2 m \delta l^{2}\right)\right] .
\end{aligned}
$$




$$
\begin{gathered}
A_{2}=C_{0}^{4}\left[\Omega^{6}\left(1+2 \delta+2 m \delta+m \delta^{2}\right)-\Omega^{4}\left\{\left(1+m+2 \delta+8 m \delta+2 m^{2} \delta\right.\right.\right. \\
\left.+2 m \delta^{2}+2 m^{2} \delta^{2}\right)+R^{2}\left(1+m^{2}+2 \delta l^{2}+2 m \delta+2 m^{2} \delta+2 m^{3} \delta l^{2}\right. \\
\left.\left.+m \delta^{2} l^{2}+m^{3} \delta^{2} l^{2}\right)\right\}+\Omega^{2}\left\{m \delta\left(4+4 m+\delta+2 m \delta+m^{2} \delta\right)\right. \\
+R^{2}\left(l^{2}+m+m^{2}+m^{3} l^{2}+4 m \delta l^{2}+m \delta+m \delta l^{2}+4 m^{2} \delta\right. \\
\left.+m^{3} \delta+5 m^{3} \delta l^{2}+2 m^{2} \delta^{2} l^{2}+2 m^{3} \delta^{2} l^{2}\right)+R^{4} m^{2}\left(1+2 \delta l^{2}\right. \\
\left.\left.\left.+2 m \delta l^{2}+m \delta^{2} l^{4}\right)\right\}-R^{2} l^{2} m^{2}\left\{4 \delta(1+m)+R^{2}\left(1+m+2 m \delta\left(1+l^{2}\right)\right)\right\}\right] . \\
A_{3}=-C_{0}^{4} u_{e}^{2}\left[\Omega^{6}(1+m+2 m \delta)-\Omega^{4}\left\{2 m(1+\delta+m \delta)+R^{2}\left(l^{2}+m+m^{2}\right.\right.\right. \\
\left.\left.+l^{2} m^{3}+2 m \delta l^{2}+2 m^{3} \delta l^{2}\right)\right\}+\Omega^{2} R^{2} m l^{2}\left\{2 \left(1+m^{2}+m \delta\right.\right. \\
\left.\left.+m^{2} \delta\right)+R^{2} m\left(1+m+2 m \delta l^{2}\right)\right\}-2 m^{3} R^{4} l^{4} . \\
A_{4}=C_{0}^{4} u_{e}^{4} \Omega^{2} m\left(\Omega^{2}-R^{2} l^{2}\right)\left(\Omega^{2}-R^{2} m^{2} l^{2}\right)=A_{4 i} .
\end{gathered}
$$

The extremely lengthy and not altogether straightforward manipulations needed in obtaining (26) and (27) are omitted here for the sake of brevity. Note that $\delta=10^{-8}$. Since $m$, the ratio of the electron to the ion mass is of the order $10^{-4}$, it is evident that $\delta$ is of the order $m^{2}$. With this knowledge it is clear that the coefficients of all the powers of $\Omega$ in (27) are in the form of a power series in $m$. It is seen from (26) and (27) that the dispersion equation (26) is a quartic in $V^{2}$ and a cubic in $\Omega^{2}$. Nevertheless it was found to be advantageous to solve (26) for $V$ rather than for $\Omega$.

It is proposed to solve (26) under the assumption (25). In almost all practical situations and particularly for the case of the ionosphere, (25) holds good and hence the following study is not restrictive from a practical point of view. In view of (25), it is legitimate to neglect $m, \delta$, and $R^{2} m$ in comparison with unity and to omit $\delta$ in comparison with $R^{2}$.

The dispersion as obtained from (26) is found to vary rapidly in the neighborhood of certain frequencies for propagation directions close to both along and across the static magnetic field. It is therefore desirable to study first the dispersion relations in the interior region which excludes both the axial $\left(1-l^{2} \gg \delta\right)$ and the transverse $\left(l^{2} \gg m\right)$ boundary layers. The investigation of the dispersion relations for the axial and the transverse boundary layers will form the subject of a subsequent paper.

The solution of the fairly complicated dispersion equation (26) is considerably simplified by the following perturbation procedure. If $V$ of the order $C_{0}$ or higher is only sought, it is found from (26) and (27), the leading terms are of the order $C_{0}^{8}$ and the other terms are lower by a factor of at least $\delta$. On retaining only the leading terms, the following simplified dispersion equation is obtained:

$$
A_{0 i} V^{4}+A_{1 i} V^{2}+A_{2 i}=0
$$

where

$$
\begin{aligned}
& A_{0 i}=\left(\Omega^{2}-1\right)\left[\Omega^{4}-\Omega^{2}\left(2+R^{2}\right)+1\right] \\
& =\left(\Omega^{2}-1\right)\left(\Omega^{2}-\Omega_{1}^{2}\right)\left(\Omega^{2}-\Omega_{2}^{2}\right) \\
& A_{1 i}=-C_{0}^{2}\left[2 \Omega^{6}-2 \Omega^{4}\left(2+R^{2}\right)+\Omega^{2}\left\{2+R^{2}\left(1+l^{2}\right)\right\}-R^{2} m\left(1+l^{2}\right]\right.
\end{aligned}
$$




$$
\begin{aligned}
A_{2 i} & =C_{0}^{4}\left[\Omega^{6}-\Omega^{4}\left(1+R^{2}\right)+\Omega^{2} R^{2}\left(l^{2}+m\right)-R^{4} l^{2} m^{2}\right] \\
& =C_{0}^{4}\left(\Omega^{2}-\Omega_{3 i}^{2}\right)\left(\Omega^{2}-\Omega_{4 i}^{2}\right)\left(\Omega^{2}-\Omega_{5 i}^{2}\right) \\
\Omega_{3 i}^{2} & =\frac{1+R^{2}}{2} \mp \sqrt{\left(\frac{1+R^{2}}{2}\right)^{2}-R^{2}} l^{2} \\
\Omega_{5 i}^{2} & =R^{2} m^{2} .
\end{aligned}
$$

It can be easily argued that (28) is valid only when $\left|\Omega^{2}-\Omega_{3 i}^{2}\right| \gg \delta$ and $\left|\Omega^{2}-\Omega_{4 i}^{2}\right| \gg \delta$. Also, when $\Omega$ is of the order $m$ and $V$ is of the order $\sqrt{m} C_{0}$, the leading terms in (26) are of the order $m^{4} C_{0}^{8}$ and their retention gives (28) again. When $\left(\Omega^{2}-R^{2} m^{2}\right)$ is of the order $m^{4}$, the approximation (28) may be shown to be not valid. Consequently, (28) gives correctly the phase velocities of the order $\sqrt{m}$ $C_{0}$ when $\Omega$ is of the order $m$ or lower such that $\left|\Omega^{2}-R^{2} m^{2}\right| \gg m^{4}$, and the phase velocities of the order $C_{0}$ for the higher values of $\Omega$ such that $\left|\Omega^{2}-\Omega_{3 i}^{2}\right| \gg \delta$ and $\left|\Omega^{2}-\Omega_{4 i}^{2}\right| \gg \delta$.

The approximate dispersion equation (28) is the same as the one obtained from considerations of the magneto-ionic theory which neglects the electron and the ion pressures completely, and its analysis shows [Seshadri, 1964a] that there are two modes of propagation. The ordinary electromagnetic mode propagates in the two frequency ranges $0 \leqslant \Omega<\Omega_{3 i}$ and $1 \leqslant \Omega \leqslant \infty$ and the extraordinary electromagnetic mode in the three frequency ranges, $0 \leqslant \Omega<\Omega_{5 i}, \Omega_{1} \leqslant \Omega<\Omega_{4 i}$ and $\Omega_{2} \leqslant \Omega \leqslant \infty$. The frequencies $\Omega_{3 i}, \Omega_{4 i}$, and $\Omega_{5 i}$ for which the phase velocity given by (28) goes to zero are called the resonant frequencies. From the foregoing discussion, it is clear that the magneto-ionic theory does not give correctly the dispersion near the resonant frequencies. The phase velocities specified by (28) become considerably smaller than $C_{0}$ in the close neighborhood of $\Omega=\Omega_{3 i}$ and $\Omega=\Omega_{4 i}$ and very much smaller than $\sqrt{m} C_{0}$ in the close neighborhood of $\Omega=\Omega_{5 i}$. In the close neighborhood of the resonant frequencies, the first term on the left side of (28) may be neglected in comparison with the other two terms with the result

$$
V^{2}=-\frac{A_{2 i}}{A_{1 i}}
$$

The solution of (28) gives

$$
V_{0, e}^{2}=-\frac{A_{1 i}}{2 A_{0 i}} \mp \frac{1}{2 A_{0 i}} \sqrt{A_{1 i}^{2}-4 A_{0 i} A_{2 i}}
$$

The upper and the lower signs in (32) correspond to the ordinary and the extraordinary electromagnetic waves respectively. The phase velocities given by (32) and plotted in figure 4 for $R^{2}=1 / 2$ are marked $\mathrm{AB}$ with various subscripts corresponding to the various branches. For the portions of the dispersion curves marked $\mathrm{AB}$, the electron and the ion pressures play negligible roles as a result the corresponding field components are predominantly transverse in character similar to the pure electromagnetic wave.

If $V$ is of the order $u_{e}$, the leading terms on the left side of (26) are of the order $C_{0}^{4} u_{e}^{4}$ and the remaining terms are lower by a factor of at least $m$. It follows, therefore, that for obtaining $V$ of the order $u_{e}$, only the leading terms need be retained resulting in the following expression for the phase velocity:

$$
V^{2}=-\frac{A_{3 i}}{A_{2 i}}
$$


where

$$
\begin{aligned}
A_{3 i} & =-C_{0}^{4} u_{e}^{2}\left[\Omega^{6}-\Omega^{4}\left\{2 m+R^{2}\left(l^{2}+m\right)\right\}+2 m \Omega^{2} R^{2} l^{2}-2 m^{3} R^{4} l^{4}\right] \\
& =\left(\Omega^{2}-R^{2} l^{2}\right)\left(\Omega^{2}-2 m\right)\left(\Omega^{2}-R^{2} m^{2} l^{2}\right) .
\end{aligned}
$$

It may be easily verified that (33) is valid only if $\left|\Omega^{2}-\Omega_{3 i}^{2}\right|>>\delta$ and $\left|\Omega^{2}-\Omega_{4 i}^{2}\right|>>\delta$. Also it can be shown that (33) yields $V$ of the order $u_{e}$ only for $\Omega^{2}>>m$ and $\left|\Omega^{2}-R^{2} l^{2}\right|>>m$ and these inequalities further limit the range of validity of (33). For $\Omega^{2}>>m, A_{2 i}$, and $A_{3 i}$ may be simplified with the result (33) reduces to

$$
V^{2}=\frac{u_{e}^{2} \Omega^{2}\left(\Omega^{2}-R^{2} l^{2}\right)}{\left(\Omega^{2}-\Omega_{3 i}^{2}\right)\left(\Omega^{2}-\Omega_{4 i}^{2}\right)}
$$

which corresponds to a propagating mode only for $\Omega_{3 i}^{2}<\Omega^{2}<R^{2} l^{2}$ and $\Omega_{4 i}^{2}<\Omega^{2}<\infty$. Note that $\Omega_{3 i}^{2}<R^{2} l^{2}<\Omega_{4 i}^{2}$. In figure 4 , the sections of the dispersion curves specified by (33a) are marked CD with two different subscripts corresponding to the two different branches. Note that when $\Omega$ tends to infinity, $V$ specified by (33a) approaches asymptotically the value $u_{e}$.

When $V$ is of the order $\sqrt{u_{e} C_{0}}$ and $\Omega^{2}-\Omega_{3 i}^{2}$ or $\Omega^{2}-\Omega_{4 i}^{2}$ is of the order $\sqrt{\delta}$, the leading terms in (26) are of the order $u_{e}^{3} C_{0}^{5}$ and the other terms are lower by a factor of $\sqrt{\delta}$ or smaller. The retention of the leading terms alone yields:

$$
A_{1 i} V^{4}+A_{2 i} V^{2}+A_{3 i}=0
$$

whose solutions are given by

$$
V^{2}=-\frac{A_{2 i}}{2 A_{1 i}} \pm \sqrt{\left(\frac{A_{2 i}}{2 A_{1 i}}\right)^{2}-\frac{A_{3 i}}{A_{1 i}}}
$$

It may be easily proved that $A_{3 i} / A_{1 i}<0$ for $\Omega$ in the close neighborhood of either $\Omega_{3 i}$ or $\Omega_{4 i}$. Hence only the upper sign in (36) will correspond to real values of $V$ and consequently, the lower sign in (36) may be disregarded.

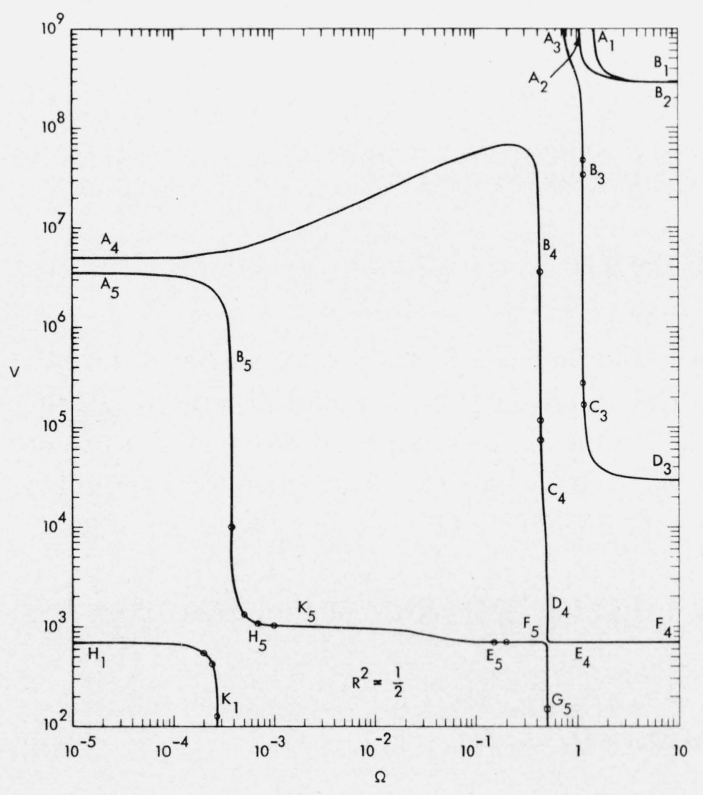

FIGURE 4. Dispersion relations for propagation at an arbitrary angle to the direction of the static magnetic field $\left(\mathrm{R}^{2}=\frac{1}{2}, \mathrm{l}^{2}=\frac{1}{2}\right)$. 
The phase velocities for $\Omega^{2}=\Omega_{3 i}^{2}$ and $\Omega^{2}=\Omega_{4 i}^{2}$ are obtained from (36) to be given by

$$
V^{2}=\sqrt{-\frac{A_{3 i}}{A_{1 i}}}
$$

With the help of (29b) and (34), (37) is seen to yield real values for $V$ of the order of magnitude $\sqrt{u_{e} C_{0}}$ for $\Omega^{2}=\Omega_{3 i}^{2}$ and $\Omega^{2}=\Omega_{4 i}^{2}$. Consequently, (37) with the upper sign, correctly gives the phase velocities when $\left(\Omega^{2}-\Omega_{3 i}^{2}\right)$ or $\left(\Omega^{2}-\Omega_{4 i}^{2}\right)$ is of the order of magnitude $\sqrt{\delta}$.

When $\left(\Omega^{2}-\Omega_{3 i}^{2}\right) \gg \sqrt{\delta}$ or $\left(\Omega^{2}-\Omega_{4 i}^{2}\right) \gg \sqrt{\delta}$, it is found with the help of $(29 \mathrm{~b}, \mathrm{c})$ and (34) that $A_{3 i} / A_{1 i}$ is of the order $\delta$ lower than $\left(A_{2 i} / A_{1 i}\right)^{2}$. The square root in (36) may therefore be expanded. Also since for $\left(\Omega^{2}-\Omega_{3 i}^{2}\right) \gg \sqrt{\delta}$ or $\left(\Omega^{2}-\Omega_{4 i}^{2}\right) \gg \sqrt{ } \delta, A_{1 i}$ and $A_{2 i}$ are of the same sign, (36) may be

shown to yield

$$
V^{2}=-\frac{A_{3 i}}{A_{2 i}}
$$

When $\left(\Omega^{2}-\Omega_{3 i}^{2}\right) \ll \sqrt{\delta}$ or $\left(\Omega^{2}-\Omega_{4 i}^{2}\right) \ll \sqrt{\delta}, A_{1 i}$ and $A_{2 i}$ are of opposite signs and (36) may then be shown to reduce to

$$
V^{2}=-\frac{A_{2 i}}{A_{1 i}}
$$

With the help of (39) and (31) the phase velocity specified by (36) is found to merge with that given by (32) for $\Omega^{2}-\Omega_{3 i}^{2} \ll \sqrt{\delta}$ or $\Omega^{2}-\Omega_{4 i}^{2} \ll \sqrt{\delta}$. In a similar manner, a comparison of (38) with (33) shows that the phase velocity given by (36) merges with that specified by (33) for $\Omega^{2}-\Omega_{3 i}^{2}$ $\gg \sqrt{\delta}$ or $\Omega^{2}-\Omega_{4 i}^{2} \gg \sqrt{\delta}$.

The phase velocities computed from (36) with the upper sign are also plotted in figure 4 and the corresponding sections of the dispersion curves are marked BC with two different subscripts corresponding to the two different branches. Note that the curve marked $A_{3} B_{3} C_{3} D_{3}$ forms one continuous dispersion curve.

When $V$ is of the order $\sqrt{m} u_{e}, \Omega \gg m$ and $\left|\Omega^{2}-R^{2} l^{2}\right| \gg m$, the retention in (26) of only the leading terms, which are of the order $C_{0}^{4} u_{e}^{4} m$, yields

$$
V^{2}=-\frac{A_{4 i}}{A_{3 i}}
$$

When $\Omega \gg m,(40)$ may be simplified with the help of (27e) and (34) to yield

$$
V^{2}=m u_{e}^{2} \Omega^{2} /\left(\Omega^{2}-2 m\right) .
$$

Obviously (41) gives real values for $V$ only for $\Omega^{2}>2 m$. Further, when $\Omega^{2}-2 m$ is of the order $m^{2}$, $V$ given by (41) becomes of the order $u_{e}$ with the result the validity of (40) is ensured only under the additional restriction that $\Omega^{2}-2 m \gg m^{2}$. The phase velocities as computed from (40) are also plotted in figure 4. Note that (41) is independent of $R$. There are two branches corresponding to the following frequency ranges: (i) $2 m<\Omega^{2}<R^{2} l^{2}$ and (ii) $R^{2} l^{2}<\Omega^{2}<\infty$ and these are respectively marked $E_{5} F_{5}$ and $E_{4} F_{4}$.

Using arguments similar to above, it can be shown that for $\left|\Omega^{2}-R^{2} l^{2}\right|$ of the order of $m, V$ is of the order $\sqrt{m} u_{e}$ and is specified by

$$
A_{2 i} V^{4}+A_{3 i} V^{2}+A_{4 i}=0
$$


The solutions of (42) are given by

$$
V_{ \pm}^{2}=-\frac{A_{3 i}}{2 A_{2 i}} \pm \sqrt{\left(\frac{A_{3 i}}{2 A_{2 i}}\right)^{2}-\frac{A_{4 i}}{A_{2 i}}}
$$

In the close neighborhood of $\Omega^{2}=R^{2} l^{2}$, it can be shown that

$$
\begin{aligned}
& \frac{A_{3 i}}{A_{2 i}}=-\frac{u_{e}^{2}\left[\Omega^{4}-\Omega^{2}\left\{2 m+R^{2} l^{2}+R^{2} m\right\}+2 m R^{2} l^{2}\right]}{\Omega^{2}\left(\Omega^{2}-R^{2}\right)} \\
& \frac{A_{4 i}}{A_{2 i}}=\frac{m u_{e}^{4}\left(\Omega^{2}-R^{2} l^{2}\right) .}{\left(\Omega^{2}-R^{2}\right)} .
\end{aligned}
$$

When $\Omega^{2}-R^{2} l^{2}$ is of the order $m$ and $R^{2}>\Omega^{2}>R^{2} l^{2}$, it is clear from (44b) that $A_{4 i} / A_{2 i}<0$. Hence, only $V_{+}$given by (43) will be real. When $\Omega^{2}-R^{2} l^{2} \gg m, A_{4 i} / A_{2 i}$ is smaller than $\left(A_{3 i} / 2 A_{2 i}\right)^{2}$ by a factor of $m$ and the square root in (43) may be expanded. For $\Omega^{2}-R^{2} l^{2} \gg m$ and $\Omega^{2}<R^{2}, A_{3 i} / A_{2 i}>0$ and (43) may be then shown to reduce to

$$
V_{+}^{2}=-\frac{A_{4 i}}{A_{3 i}}=\frac{m u_{e}^{2} \Omega^{2}}{\left(\Omega^{2}-2 m\right)} .
$$

Comparison of (45) with (41) shows that $V_{+}$given by (43) merges with the phase velocity given by (41) for $\Omega^{2}-R^{2} l^{2} \gg m$. When $2 m<R^{2} l^{2}, A_{4 i} / A_{2 i}>0$ and $A_{3 i} / A_{2 i}<0$. Hence $V_{+}$and $V_{-}$given by (43) are both real. If $R^{2} l^{2}-\Omega^{2} \gg m, A_{3 i} / A_{2 i}<0$ and with the help of (43), it can be shown that

$$
V_{+}^{2}=-\frac{A_{3 i}}{A_{2 i}}
$$

and

$$
V_{-}^{2}=-\frac{A_{4 i}}{A_{3 i}}=\frac{m u_{e}^{2} \Omega^{2}}{\left(\Omega^{2}-2 m\right)} .
$$

An inspection of (33) and (46a) shows that $V_{+}$given by (43) merges with that given by (33) for $R^{2} l^{2} \gg m$. Similarly, it is seen that $V_{-}$given by (43) merges with that given by (40) and (41) for $R^{2} l^{2}-\Omega^{2} \gg m$. Also, when $\Omega^{2}=R^{2} l^{2}, A_{4 i} / A_{2 i}=0$ and $A_{3 i} / A_{2 i}<0$. Hence, (43) yields

$$
V_{+}^{2}=-\frac{A_{3 i}}{A_{2 i}} ; V_{-}^{2}=0 \text { for } \Omega^{2}=R^{2} l^{2}
$$

The phase velocities, $V_{+}$and $V_{-}$given by (43) and plotted in figures 4 are marked $D_{4} E_{4}$ and $F_{5} G_{5}$ respectively. From the foregoing arguments, it is clear that the section of the dispersion curve marked $D_{4} E_{4}$ merges continuously and smoothly with that marked $C_{4} D_{4}$ on the low-frequency side and with the section $E_{4} F_{4}$ on the high frequency side with the result the sections marked $A_{4} B_{4}$, $B_{4} C_{4}, C_{4} D_{4}, D_{4} E_{4}$ and $E_{4} F_{4}$ together form one smooth curve. Also the section $F_{5} G_{5}$ of the dispersion

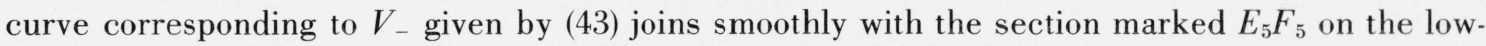
frequency side. Note that this curve goes to zero for $\Omega=R l$.

When $\Omega$ is of the order $m$, the phase velocity of the order $\sqrt{m} u_{e}$ may be shown to be specified by

$$
V^{2}=-\frac{A_{3 i}}{A_{2 i}}
$$

With the help of (29c) and (34), (48) may be simplified to yield for $\Omega$ of the order of $m$

$$
V^{2}=2 m u_{e}^{2} \frac{\left(\Omega^{2}-R^{2} l^{2} m^{2}\right)}{\left(\Omega^{2}-R^{2} m^{2}\right)} .
$$


Only for $0<\Omega<R l m$ and $\Omega>R m$, does (49) yield real values for $V$. Note that $V$ specified by (49) attains the value $\sqrt{2 m} u_{e} l$ for $\Omega$ tending to zero and goes to zero for $\Omega=R l m$. It may be proved that (49) is valid even in the close neighborhood of $\Omega=R l m$. When $\left|\Omega^{2}-R^{2} m^{2}\right|$ is of the order $m^{3}, V$ given by (49) becomes of the order $u_{e}$ and hence (49) is not valid. The phase velocity, as obtained from (49) is also plotted in figures 4 and the corresponding sections of the dispersion curves are marked by $H_{1} K_{1}$ and $H_{5} K_{5}$ respectively.

When $\left|\Omega^{2}-R^{2} m^{2}\right|$ is of the order $m^{3}, V$ is of the order $u_{e}$ and it may be shown to be specified by

$$
\tilde{A}_{1 i} V^{4}+\tilde{A}_{2 i} V^{2}+\tilde{A}_{3 i}=0
$$

where

$$
\begin{gathered}
\tilde{A}_{1 i}=m\left(1+l^{2}\right) \\
\tilde{A}_{2 i}=C_{0}^{2}\left[\Omega^{2}\left(l^{2}+m\right)-R^{2} l^{2} m^{2}\right] \\
\tilde{A}_{3 i}=-2 C_{0}^{2} u_{e}^{2} l^{2} m\left(\Omega^{2}-R^{2} l^{2} m^{2}\right) .
\end{gathered}
$$

The two solutions of (50) are given by

$$
V_{1,2}^{2}=-\frac{\tilde{A_{2 i}}}{2 \widetilde{A_{1 i}}} \pm \sqrt{\left(\frac{\tilde{A_{2 i}}}{2 \widetilde{A_{1 i}}}\right)^{2}-\frac{\widetilde{A_{3 i}}}{\widetilde{A_{1 i}}}}
$$

where $V_{1}$ and $V_{22}$ correspond to the upper and the lower signs in (52) respectively. For $\Omega^{2}>R^{2} m^{2}$, $\tilde{A}_{3 i} \mid \tilde{A}_{1 i}<0$ and hence only $V_{1}$ will be real. For $\Omega^{2}-R^{2} m^{2} \gg m^{3},\left(\tilde{A_{2 i}} / 2 \tilde{A}_{1 i}\right)^{2} \gg\left|\tilde{A}_{3 i}\right| \tilde{A}_{1 i} \mid$ and $\tilde{A_{1 i}} \mid \tilde{A_{2 i}}$ $>0$; hence, (52) yields for $V_{1}^{2}$

$$
V_{1}^{2}=-\frac{\tilde{A}_{3 i}}{\tilde{A}_{2 i}}=2 m u_{\rho}^{2} \frac{\left(\Omega^{2}-R^{2} l^{2} m^{2}\right)}{\left(\Omega^{2}-R^{2} m^{2}\right)},
$$

which is the same as that given by (48) and (49) showing that the phase velocity curve for $V_{1}$ specified by (52) merges with that given by (48) for $\Omega^{2}-R^{2} m^{2} \gg m^{3}$.

For $R^{2} l^{2} m^{2}<\Omega^{2}<R^{2} m^{2}, A_{3 i} / A_{1 i}<0$ and hence again only $V_{1}$ is real. Also if $R^{2} m^{2}-\Omega^{2} \gg m^{3}$, $\tilde{A_{1 i}} \mid \tilde{A_{2 i}}<0$ and therefore, (52) may be simplified to yield

$$
V_{1}^{2}=-\frac{\tilde{A_{2 i}}}{\widetilde{A_{1 i}}}
$$

which is seen to be the same as (31). As a result, it follows that the phase velocity curve for $V_{1}$ specified by (52) merges with that for $V_{e}$ given by (32).

If $\Omega^{2}<R^{2} l^{2} m^{2}, \widetilde{A_{3 i}} / \widetilde{A_{1 i}}>0, \widetilde{A_{2 i}} / \widetilde{A_{1 i}}<0$, and hence both $V_{1}$ and $V_{22}$ are real. From (51) and (52), it is easily deduced that

$$
V_{1}^{2}=-\frac{\widetilde{A_{2 i}}}{\widetilde{A_{1 i}}}
$$

and

$$
V_{2}^{2}=-\frac{\tilde{A_{3 i}}}{\widetilde{A_{2 i}}}=2 m u_{e}^{2} \frac{\left(\Omega^{2}-R^{2} l^{2} m^{2}\right)}{\left(\Omega^{2}-R^{2} m^{2}\right)} .
$$

Clearly (55) shows that $V_{2}$ specified by (52) is real and identical with that given by (48). The phase velocity curves computed from (52) are sketched in figure 4 . The curve corresponding to $V_{2}$ is the same as the one marked $H_{1} K_{1}$ and that corresponding to $V_{1}$ is marked $B_{5} H_{5}$. From what has been 
stated before, it is clear that the section $B_{5} H_{5}$ joins smoothly with the section $A_{5} B_{5}$ on the lower frequency side and with the section $H_{5} K_{5}$ on the higher frequency side.

Following the same procedure employed before, it can be easily deduced that when $\Omega^{2}$ is of the order $m ; V$ of the order $\sqrt{m} u_{e}$ is specified by the following equation:

$$
\bar{A}_{2 i} V^{4}+\bar{A}_{3 i} V^{2}+\bar{A}_{4 i}=0 \text {. }
$$

For $\Omega^{2}$ of the order $m$, the coefficients in (56) may be readily shown with the help of (29c), (34), and (27e) to be given by

$$
\begin{aligned}
& \bar{A}_{2 i}=1 \\
& \overline{A_{3 i}}=u_{e}^{2}\left(\Omega^{2}-2 m\right) \\
& \bar{A}_{4 i}=-m u_{e}^{4} \Omega^{2} .
\end{aligned}
$$

The two solutions of (56) are

$$
V_{3,4}^{2}=-\frac{\bar{A}_{3 i}}{2 \bar{A}_{2 i}} \pm \sqrt{\left(\frac{\bar{A}_{3 i}}{2 \bar{A}_{2 i}}\right)^{2}-\frac{\bar{A}_{4 i}}{\bar{A}_{2 i}}}
$$

Since $A_{4 i} / A_{2 i}<0$, only $V_{3}$ corresponding to the upper sign in (58) is real. It follows from (58) that

$$
V_{3}^{2}=2 m u_{e}^{2} \text { for } \Omega^{2} \ll 2 m
$$

and

$$
=\frac{m u_{e}^{2} \Omega^{2}}{\left(\Omega^{2}-2 m\right)} \text { for } \Omega^{2} \gg 2 m \text {. }
$$

It is seen from (41) and (59b) that the phase velocity curve for $V_{3}$ given by (58) merges with that specified by (41) for $\Omega^{2} \gg 2 m$. For $\Omega^{2}$ of the order $m$, (49) becomes identical to (59a) showing that the phase velocity curve for $V_{3}$ specified by (58) for $\Omega^{2} \ll 2 m$ merges with that given by (49). The phase velocity curve for $V_{3}$, as obtained from (58) and plotted in figure 4 is marked $K_{5} E_{5}$. It is to be noted that the section of the dispersion curve marked $K_{5} E_{5}$ joins smoothly with the section $H_{5} K_{5}$ on the lower frequency side and with the section $E_{5} F_{5}$ on the higher frequency side. Also in the close neighborhood of $\Omega^{2}=2 m, V$ changes rather rapidly from the value $\sqrt{2 m} u_{e}$ which is equal to the acoustic velcoity in the gas of both the charged particles to the value $\sqrt{m} u_{e}$ which is equal to the acoustic velocity in the ion gas alone. This feature is the same as obtained for an isotropic two component plasma [Seshadri, 1965]. Further, it follows that the various sections of the dispersion curves marked respectively by $A_{5} B_{5}, B_{5} H_{5}, H_{5} K_{5}, K_{5} E_{5}, E_{5} F_{5}$, and $F_{5} G_{5}$ form one smooth curve.

It is of interest to examine the dispersion for extremely low frequencies such that $\Omega^{2} \ll R^{2} m^{2}$. For this case, (26) may be simplified to yield

$$
V^{8}-R^{2} m C_{0}^{2}\left(1+l^{2}\right) V^{6}+R^{4} l^{2} m^{2} C_{0}^{4} V^{4}-2 m^{3} R^{4} l^{4} C_{0}^{4} u_{e}^{2} V^{2}-C_{0}^{4} u_{e}^{4} \Omega^{2} m^{3} R^{4} l^{4}=0 .
$$

Using arguments similar to those given previously, it follows from (60) that $V$ of the order $\sqrt{m} C_{0}$ is given by the following equation:

$$
V^{4}-R^{2} m C_{0}^{2}\left(1+l^{2}\right) V^{2}+R^{4} l^{2} m^{2} C_{0}^{4}=0 .
$$


The solutions of (61) are

$$
V^{2}=R^{2} m C_{0}^{2}=V_{\alpha}^{2} ; \quad V^{2}=R^{2} m C_{0}^{2} l^{2}
$$

Also the phase velocities of the order $\sqrt{m} u_{e}$ are obtained from (60) to be given by

$$
V^{2}=2 m l^{2} u_{e}^{2}
$$

It follows therefore that in the low-frequency limit there are only three propagating modes with the phase velocities specified by (62) and (63). It is easily recognized that the first part of the section $A_{4} B_{4}$ of the dispersion curves in figure 4 corresponds to the fast hydromagnetic wave whose phase velocity is equal to the Alfvèn wave velocity $V_{\alpha}=C_{0} R \sqrt{m}$ for all directions of propagation. The mode denoted by the first part of the section $A_{5} B_{5}$ of the dispersion curve in figure 4 is the slow hydromagnetic wave with a phase velocity which is equal to the Alfvèn wave velocity in the direction of the static magnetic field $B_{0}$ and which changes by a factor of $\cos \theta$ for the other directions of propagation. The mode denoted by $H_{1} K_{1}$ in figure 4 is the sound wave whose velocity in the direction of $B_{0}$ is equal to the acoustic velocity $\sqrt{2 m} u_{e}$ in the entire gas of charged particles. The phase velocity of the sound wave, just like the slow hydromagnetic wave, changes by a factor of $\cos \theta$ for the other directions of propagation. The spatial dispersion of the three modes in the low frequency limit, as obtained here, is in accordance with the results obtained previously from magnetohydrodynamic considerations [Seshadri, 1964a].

The section of the dispersion curve denoted by $K_{5} E_{5}$ for $\Omega^{2}$ considerably less than $2 m$, has a phase velocity equal to the acoustic velocity in the entire gas of charged particles for all directions of propagation except in the transverse boundary layer. The static magnetic field has very little effect on the dispersion for this section of the phase velocity curve.

Only the identification of the various modes given in figure 4 remain. In a two fluid compressible plasma there are only four independent modes of oscillation, and these are designated as follows: (i) the modified extraordinary electromagnetic mode $\operatorname{MEM}(X)$, (ii) the modified ordinary electromagnetic mode, MEM(0), (iii) the modified electron plasma mode, MEP and (iv) the modified ion plasma mode, MIP. The branch $A_{1} B_{1}$ of the dispersion curve corresponds to the $\operatorname{MEM}(X)$ mode, since its cutoff depends on the strength $B_{0}$ of the external magnetic field and since, in the limit of infinite frequency, its phase velocity approaches $C_{0}$. The branch $A_{2} B_{2}$ corresponds to the $\operatorname{MEM}(0)$ mode, since its cutoff is independent of $B_{0}$ and since, in the limit of infinite frequency, the phase velocity of this mode also approaches $C_{0}$. The branch $A_{3} B_{3} C_{3} D_{3}$ belongs to the MEP mode since, in the limit of infinite frequency, its phase velocity approaches the acoustic velocity $u_{e}$ in the electron gas. The branch $A_{4} B_{4} C_{4} D_{4} E_{4} F_{4}$ belongs to the MIP mode for, in the limit of infinite frequency, its phase velocity becomes equal to the acoustic velocity $u_{i}$ in the ion gas. The remaining two branches of the dispersion curves do not extend to infinite frequency and are obviously parts of the above mentioned four modes. It may be easily argued that the branch $A_{5} G_{5}$ is a part of the $\operatorname{MEM}(X)$ mode. Also, it is appropriate to associate the branch $H_{1} K_{1}$ with the MEP mode.

In an isotropic plasma, two out of the four possible modes are purely longitudinal and the other two are purely transverse. But in an anisotropic plasma, all the four modes contain both longitudinal and transverse field components, and for the purpose of distinguishing these from the corresponding ones of the isotropic plasma, the word 'modified' is attached to their designation. Although the modes of anisotropic plasma, strictly speaking, contain both longitudinal and transverse field components, the procedure used in the analysis of the dispersion uncovers the fact that these modes are predominantly longitudinal in certain frequency ranges and predominantly transverse in the other ranges of frequency. The MEM(0) mode is predominantly transverse in its entire range of propagation, $1<\Omega<\infty$. The $\operatorname{MEM}(X)$ mode is predominantly transverse for $0<\Omega<R m$ and $\Omega_{2}<\Omega<\infty$ and is predominantly longitudinal for $R m<\Omega<R l$. The MEP mode is predominantly longitudinal for $0<\Omega<R m l$, and $\Omega_{4 i}<\Omega<\infty$ is predominantly transverse for $\Omega_{1}<\Omega<\Omega_{4 i}$. The MIP mode is predominantly transverse in the frequency range $0<\Omega<\Omega_{3 i}$ 
and is predominantly longitudinal for $\Omega_{3 i}<\Omega<\infty$. It is evident from the above discussion that in the neighborhood of the three frequencies $\Omega=R m, \Omega=\Omega_{3 i}$ and $\Omega=\Omega_{4 i}$, coupling of the transverse and the longitudinal type waves takes place.

\section{Concluding Remarks}

It is desirable to contrast the treatment of the plane wave propagation in a two component warm plasma contained in this paper with those aspects of the problem that are discussed in the books by Stix [1962], Allis, Buchsbaum, and Bers [1963] and Denisse and Delcroix [1963]. Apart from the careful consideration of the characteristics of plane wave propagation in a warm plasma through the use of Boltzmann equations, Stix [1962] has discussed the dispersion of the low frequency ion acoustic wave and the ion cyclotron wave. The low-frequency ion acoustic wave corresponds to the first half of the branch $H_{1} K_{1}$ of the dispersion curve, and the ion cyclotron wave consists of two parts, the first part corresponds to the branch $B_{5} H_{5}$ and the second part to the second half of the branch $H_{1} K_{1}$. Since he treated only the low phase velocity and low-frequency approximation, Stix obtained a gap in the frequency spectrum in the range $R l<\Omega<R$. Note however that in this frequency gap, the two Alfvén waves propagate.

A parametric representation was first introduced by Clemmow and Mullaly [1955] for ascertaining the regions of propagation of the possible plane wave modes in an unbounded and homogeneous magneto-ionic medium. The two parameters used by Clemmow and Mullaly [1955] are $X=\omega_{p e}^{2} / \omega^{2}$ and $Y^{2}=\omega_{c e}^{2} / \omega^{2}$. In the analysis of the properties of wave propagation in a plasma, the determination of the propagation characteristics explicitly as a function of the wave frequency $\omega$ is almost always desired. Such a determination is difficult if the parameters $X$ and $Y^{2}$ are used, since the wave frequency is mixed in both of them. Therefore, in the present analysis, instead of the conventional parameters, the following normalized frequencies are used:

$$
\Omega=\frac{\omega}{\omega_{p e}} ; R=\frac{\omega_{c e}}{\omega_{p e}} .
$$

Allis, Buchsbaum, and Bers [1963] have considerably extended the Clemmow-Mullaly plots, for example, to apply to a two component plasma. The two parameters employed by Allis, Buchsbaum, and Bers are

$$
\alpha^{2}=\frac{\omega_{p e}^{2}+\omega_{p i}^{2}}{\omega^{2}} \quad \beta^{2}=\frac{\omega_{c e} \omega_{c i}}{\omega^{2}}
$$

It is to be noted that $\alpha^{2}$ is proportional to the number density and $\beta^{2}$ is proportional to the square of the external static magnetic field. Note also that both the parameters $\alpha^{2}$ and $\beta^{2}$, also contain the wave frequency. In order to overcome certain practical difficulties encountered in the construction of the graphical plots, Allis, Buchsbaum, and Bers [1963] use the assumed mass ratio $m=m_{e} / m_{i}=\frac{1}{3}$ between an electron and an ion. Further, as the mass ratio vanishes, the parameter $\beta^{2}$ also vanishes. To avoid the above-mentioned practical difficulties, the graphical plots were constructed (but not included in this paper for the sake of brevity) in terms of the two parameters $\Omega^{2}$ and $R^{2}$. A discussion of this new diagrammatic representation may be found in Seshadri [1964b] for a single component warm plasma and in Seshadri [1964a] for a two component cold plasma. This new parametric representation has two special features, namely, (i) the wave frequency $\omega$ is contained only in one of the parameters and (ii) the mass ratio $m$ does not appear in either of the parameters. The first feature facilitates greatly the study of the propagation characteristics explicitly as a function of frequency and the second feature enables the plots to be used with equal facility for both the single and the two component plasma, with the result it is possible to ascertain from the graphical plots the influence of the heavy ion motion on the propagation characteristics of a plane wave in a plasma. Moreover the plots in the $\Omega^{2}-R^{2}$ parameter space can be employed as is for a multicomponent plasma. 
Allis, Buchsbaum, and Bers [1963] have noted that the plane wave dispersion relation with electron and ion thermal motion included is sufficiently involved that no simple form has been found for it. Consequently, their discussion of the plane wave dispersion relations in a two component warm plasma proceeds by first neglecting the coupling of the electromagnetic and the plasma waves and the coupling being then described intuitively on the basis of the understanding of the corresponding coupling in a single component warm plasma.

Denisse and Delcroix [1963] have considered various aspects of the characteristics of plane wave propagation in a two component warm plasma. For the case of propagation along and across the external magnetic field, they have plotted the dispersion curves in terms of $x^{\prime}$ and $y^{\prime}$ where $x^{\prime}=\log \left[\left(\omega_{p e}^{2}+\omega_{p i}^{2}\right) / \omega^{2}\right]$ and $y^{\prime}=C_{0}^{2} / V^{2}$ for $\left|C_{0}^{2} / V^{2}\right| \leqslant 1$ and $y^{\prime}=1+\log \left(1+\log \left|C_{0}^{2} / V^{2}\right|\right)$ for $\left|C_{0}^{2} / V^{2}\right| \geqslant 1$. In view of the different scales adopted for the different phase velocity regions and in view of the $\log \log$ scale adopted for the low phase velocity region, a clear overall picture of the variation of the basic quantity, namely the phase velocity with the frequency, as is depicted in figures 2 and 3 , is difficult to obtain from the work of Denisse and Delcroix. For the general case of propagation at an arbitrary angle to the direction of the static magnetic field, Denisse and Delcroix have attempted to extricate the rules governing the propagation of the coupled longitudinal and transverse modes. The dispersion relation has been expressed both as a quartic in $u\left[=\left(C_{0}^{2} / V^{2}-1\right)^{2}\right]$ and as a cubic in $x\left[=\left(\omega_{p e}^{2}+\omega_{p i}^{2}\right) / \omega^{2}\right]$. They have examined the vertical and the horizontal asymptotes corresponding to $x$ going to zero and infinity and thus have obtained respectively the limiting behavior of the dispersion for infinite and zero frequencies. They have not examined the dispersion over the whole frequency region nor are any dispersion diagrams for the arbitrary direction of propagation given.

In contrast to the treatments contained in the books by Allis, Buchsbaum, and Bers [1963] and Denisse and Delcroix [1963], in this paper the dispersion equation for the arbitrary direction of propagation of the plane wave has been cast in a rather simplified form as given in (26) and (27). By systematically analyzing the dispersion equation in the various regimes of frequency and phase velocity, it has been possible not only to treat the coupling of the transverse and the longitudinal waves, but also to obtain simple analytical expressions for the dispersion relations in the various intervals of frequency and phase velocity, as well as for the frequencies where the transverse and the longitudinal type waves couple. Also, the dispersion curves are plotted in terms of the phase velocity as a function of frequency for a typical case in which the propagation vector makes an angle of $45 \mathrm{deg}$ with the direction of the static magnetic field.

In conclusion, it is appropriate to sum up that the plane wave dispersion relations in a two component warm plasma are worked out in this paper for the general case of propagation at an arbitrary angle to the direction of the static magnetic field. This treatment does not cover the transition regions corresponding to propagation vectors in the close neighborhood of either the direction of the magnetostatic field or that perpendicular to it. The treatment of the dispersion relations for the axial and the transverse boundary layers is reserved for a subsequent paper. Once the dispersion is known for all angles including the axial and the transverse transition regions, it will be possible to depict the spatial dispersion, that is, the variation of the phase velocity as a function of angle for various typical frequencies, as was done for the two component cold plasma [Seshadri, 1964a].

The author is grateful to Ronald V. Row for the many helpful discussions and to P. A. Kimball for the valuable assistance with the numerical computations.

This research was supported by the Office of Naval Research under Contract Nonr-3185(00). 


\section{References}

Allis, W. P., S. J. Buchsbaum, and A. Bers (1963), Waves in anisotropic plasmas, (MIT Press, Cambridge, Mass.).

Astrom, E. (1950), Magnetohydrodynamic waves in a plasma, Nature 165, 1019-1020.

Astrom, E. (1951), On waves in an ionized gas, Arkiv. Fysik 2, No. 42, 443-457.

Booker, H. G. (1963), The magnetoionic theory at hydromagnetic frequencies, presented at the 1963 Spring URSI Meeting, April 29-May 2, Washington, D.C.

Clemmow, P.C., and R.F. Mullaly (1955), Dependence of the refractive index in magnetoionic theory on the direction of the wave normal, Physics of the Ionosphere, Report of Physical Society Conference, Cavendish Laboratory, Physical Society, London, 340-350.

Denisse, J. F., and J. L. Delcroix (1963), Plasma waves (Interscience Publishers, New York, N.Y.).

Fejer, J. A. (1960), Hydromagnetic wave propagation in the ionosphere, J. Atmospheric Terrest. Phys. 18, 135-146.

Hines, C. O. (1953), Generalized magnetohydrodynamic formulae, Proc. Camb. Phil. Soc. 49, 299-307.

Hines, C. O. (1957), Heavy-ion effects in audio-frequency radio propagation, J. Atmospheric Terrest. Phys. $11,36-42$.

Hines, C. O. (1963), The relation between hydromagnetic waves and the magnetoionic theory; Electromagnetic theory and antennas, ed. E. C. Jordan, 287-299 (Pergamon Press).

Kieburtz, R. B. (1964), VLF propagation in a compressible ionosphere, Radio Sci. J. Res. NBS/USNC-URSI 68D, No. 7, 795-805.

Oster, L. (1960), Linearized theory of plasma oscillations, Rev. Mod. Phys. 32, 141-168.

Pai, S. I. (1960), Wave motions of small amplitude in a fully ionized plasma without external magnetic field, Rev. Mod. Phys. 32, No. 4, 882-887.

Pai, S. I. (1962), Wave motions of small amplitude in a fully ionized plasma under applied magnetic field, Phys. Fluids 5, No. 2, 234-240.

Ratcliffe, J. A. (1959), The magnetoionic theory and its application to the ionosphere (Cambridge University Press, Cambridge, England).

Seshadri, S. R. (1964a), The magnetoionic theory at hydromagnetic frequencies, Research Report No. 392, Applied Research Laboratory, Sylvania Electronic Systems, Waltham, Mass.

Seshadri, S. R. (1964b), Wave propagation in a compressible ionosphere, I and II, Radio Sci. J. Res. NBS/USNC-URSI 68D, No. 12, 1285-1307.

Seshadri, S. R. (1965), Radiation from electromagnetic sources in a plasma, IEEE Trans. Ant. Prop. AP-13, No. 1.

Stix, T. H. (1957), Oscillations of a cylindrical plasma, Phys. Rev. 106, No. 6, 1146-1150.

Stix, T. H. (1952), The theory of plasma waves (McGraw-Hill Book Co., Inc., New York, N.Y.).

Storey, L. R. O. (1956), A method to detect the presence of ionized hydrogen in the outer atmosphere, Can. J. Phys. 34, 1153-1163.

Tanenbaum, B. S. (1961), Dispersion relations in a stationary plasma, Phys. Fluids 4, No. 10, 1262-1272.

Watanabe, T. (1961), Waves in a rarified ionized gas propagated transverse to an external magnetic field, Can. J. Phys. 39, No. 7, 1044-1057.

(Paper 69D4-493) 